\title{
Effect of substrate orientation on CdS homoepitaxy by molecular dynamics
}

\author{
S. Almeida ${ }^{{ }^{*}}$, J.J. Chavez ${ }^{1}$, X. W. Zhou ${ }^{2}$, and D. Zubia ${ }^{1}$ \\ ${ }^{1}$ University of Texas at El Paso, Department of Electrical and Computer Engineering, El Paso, Texas \\ 79968, USA \\ ${ }^{2}$ Sandia National Laboratories, Mechanics of Materials Department, Livermore, California 94550, USA \\ Corresponding author: ${ }^{*}$ sfalmeida@utep.edu; phone: (915)747-6580
}

\section{Abstract}

CdS homoepitaxy growth was performed by molecular dynamics using different substrate orientations and structures in order to analyze the CdS crystallinity. As anticipated from thermodynamics of homoepitaxy, highly crystalline films with only point defects were obtained on substrates with rectangular surface geometries, including [11ㄹ] zinc blende (ZB), [10 $\overline{1} 0]$ wurtzite (WZ), $[11 \overline{2} 0] \mathrm{WZ},[110] \mathrm{ZB},[010] \mathrm{ZB}$, and $\left[\frac{1}{10} 1 \frac{1}{10}\right] \mathrm{ZB}$. In contrast, films grown on substrates with hexagonal surface geometries, corresponding to the [0001] WZ and [111] ZB growth directions, showed structures with a large number of defects including; anti-sites, vacancies, stacking faults, twinning, and polytypism. WZ and ZB transitions and grain boundaries are identified using a lattice identification algorithm and represented graphically in a structural map. A dislocation analysis was performed to detect, identify, and quantify linear defects within the atomistic data. Systematic simulations using different temperatures, deposition rates, and substrate polarities were perform to analyze the trends of dislocation densities on [0001] WZ direction and showed persistent polytypism. The polytypism observed in the films grown on the substrates with hexagonal surface geometry is attributed to the similar formation energies of the WZ and ZB phases.

Keywords: A1. Molecular dynamics; A3. Homoepitaxy growth; A1. Defects; A2. Single crystal growth; B1. Cadmium sulfide.

\section{Introduction}

Cadmium sulfide (CdS) is a II-VI semiconductor material widely used in photovoltaic solar cells $[1,2]$ due to its direct band gap of $2.48 \mathrm{eV}$ and absorption coefficient of $4 \times 10^{6} \mathrm{~m}^{-1}$ (at $500 \mathrm{~nm}$ ) [3]. Additionally, CdS nanowires [4] and quantum dots [5] have attracted much attention for optical and electronic applications such as: light-emitting diodes [6], photodetectors [7], lasers [8], waveguides [9], logic circuits [10], and transistors [11]. Due to the potential of CdS to be used as a building block in future electronics, a variety of synthesis and deposition techniques have been investigated including: metal-organic chemical vapor deposition [12], physical vapor deposition (PVD) [4], electrodeposition [13], thermal evaporation [14], electrochemical synthesis [15], close space sublimation [16], and chemical bath deposition (CBD) [17].

CdS is stable in the wurtzite (WZ) and cubic zinc blende (ZB) crystal structures [18]. ZB and WZ CdS nanostructures have been reported to grow along different orientations with varying degree of crystallinity. A distinction in the degree of crystallinity emerges depending on the polarity and surface geometry. In particular, growths on polar surfaces with hexagonal geometry do not always result in highly crystalline [12], [19], [20] structures. For example, Ouyang, et al. reported that chemical deposition of CdS nanorods grown along $\langle 0001\rangle$ WZ direction were defected with stacking faults (SF), exhibiting polytypism with a mixture of ZB and WZ phases [21] similar to previous reports [22], [23], [24]. Along the ZB polar $\langle 111\rangle$ orientation, CdS quantum dots deposited via chemical co-precipitation were reported to contain SF, twinning, and polytypism by Kumar, et al. [5].

Strikingly, growths reported on surfaces with rectangular atomic surface geometry seem to indicate only highly crystalline structures. For example, along the [10 $\overline{1} 0]$ and [11 $\overline{2} 0]$ directions, single crystal 
growth of WZ nanocrystals have been reported by Berrelet, et al. [25] and Ma, et al. [26] via chemical vapor deposition (CVD). These works indicate that the geometry of the CdS surface has a strong influence on the crystallinity of the overgrown homoepitaxial CdS; however a systematic study of this influence is lacking. In this work, the homoepitaxial growth of CdS along different orientations was simulated via high fidelity molecular dynamics (MD). CdS was deposited on WZ and ZB CdS substrates with different crystal orientations. Thereafter the resultant structures were analyzed to detect, identify, and quantify linear defects and their dependence on surface geometry. Lastly, simulated growths at different deposition conditions were employed to study their influence in film crystallinity by observing dislocation density trends.

\section{MD simulation details}

A total of 8 substrates with different structures and surface orientations were created. Two of them had polar hexagonal surface symmetries corresponding to the [0001] WZ and [111] ZB growth directions. The other six substrates had polar and non-polar rectangular surface symmetries corresponding to the following growth directions: [1010] WZ, [11 $\overline{2} 0] \mathrm{WZ},[110] \mathrm{ZB},[010] \mathrm{ZB}$, and $\left[\frac{1}{10} 1 \frac{1}{10}\right]$ ZB (equivalent to a $[100]$ ZB tilted by $\sim 8^{\circ}$ representing a miscut). The simulations were performed with the Large-Scale Atomic/Molecular Massively Parallel Simulator (LAMMPS) code [27] using the Stillinger-Weber potential developed in ref. [28]. The lattice parameters were $a=4.12597 \AA$ and $\mathrm{C}=6.73768 \AA$ for the $\mathrm{WZ}$ substrates and $\mathrm{a}=5.8350 \AA$ for the $Z \mathrm{~B}$ substrates. Due to limited computational resources, a fast deposition rate of $\sim 0.48 \mathrm{~nm} / \mathrm{ns}$ was utilized to grow enough material for the analysis within tractable nanosecond windows. However, the effect of unrealistically high deposition rate is mitigated by using a high temperature of $1200 \mathrm{~K}$ to accelerate the dynamics of the growth. On the other hand, we emphasize that the objective of the MD studies is to show qualitative trends that agree with experimental results as a way to gain insights into growth mechanisms. The Nose-Hoover [29] algorithm was used to approximate isothermal growth conditions. During deposition, individual $\mathrm{Cd}$ and $\mathrm{S}$ atoms are randomly added at equal frequencies so that over time they give a 1:1 ratio of $\mathrm{Cd}$ to $\mathrm{S}$ atoms. Free boundary condition was employed in the growth direction and periodic boundary conditions in the orthogonal directions. The position of the atoms within the bottom two monolayers was fixed throughout each of the simulations to prevent crystal drift due to adatom momentum transfer. Following deposition, the film and substrate were subjected to a three step post-deposition treatment simulation. No more adatoms were added during this treatment. The first step consisted of an annealing stage in which the samples were heated at the deposition temperature for $\sim 8 \mathrm{~ns}$ in order to provide the system time and energy to find a stable configuration. During the second step the system temperature was ramped down from the deposition temperature towards $50 \mathrm{~K}$ over a period of $\sim 8 \mathrm{~ns}$. The third step involved minimizing the energy of the system (to approximate $0 \mathrm{~K}$ conditions). The last two steps reduce thermal effects (such as atomic vibration) to improve the clarity of the data and increase the accuracy of the lattice structure and defect analysis algorithms.

\section{Results and discussions}

Atomistic visualizations of the eight simulated CdS homoepitaxial growths are presented in Figures 1 and 2, which were rendered using the OVITO software tool [30]. Figure 1 contains the CdS grown on the six nonpolar surfaces with rectangular symmetry which include: $[10 \overline{1} 0] \mathrm{WZ},[11 \overline{2} 0] \mathrm{WZ}$,

[110] ZB, [010] ZB, $\left[\frac{1}{10} 1 \frac{1}{10}\right] \mathrm{ZB}$, and [112] ZB. In each case, an atomic icon representative of the surface geometry is provided as a guide. With one exception, all the nanostructures grown on the nonpolar surfaces with rectangular symmetry showed a high degree of crystallinity with only point defects being present in the form of anti-sites and vacancies; no dislocations were observed. The exception was [112] ZB; in this orientation a low density of stacking faults was observed in addition to point defects. However no twinning or polytypism were found. These results are in good agreement with experimental observations reported in the literature for rectangular surfaces. For example, growth 
in $[10 \overline{1} 0]$ WZ and $[11 \overline{2} 0]$ WZ orientations have been experimentally shown to result in single crystals [25] [26].

An additional simulation was performed which uses a reconstructed (010)-(2X1) ZB surface. The simulated growth was similar to the non-reconstructed (010) ZB surface. Since these simulations use Newton's law and interatomic potentials, the reconstructed surface is always relaxed to the lattice structure when it is buried into the bulk. Similar phenomenon has been observed experimentally [31].

Growth on two polar surfaces with hexagonal symmetry, (0001) WZ and (111) ZB, showed a large number of defects including: anti-sites, vacancies, stacking faults, twinning, and polytypism (existence of multiple phases). Atomistic visualizations of the MD simulated growths are shown in Figure 2 where twinning, stacking faults, and ZB and WZ domains are marked in red. In order to highlight the ZB and $W Z$ domains and corresponding grain boundaries, a structural analysis was performed as shown in Figure 3 using the common neighbor analysis algorithm for diamond (ZB case) and hexagonal diamond (WZ case) lattices included within OVITO [30]. In Figure 3, the atoms are color coded in order to more easily identify the different crystal domains. Red atoms indicate the WZ structure whereas blue atoms correspond to ZB. Atoms that were not recognized to be in either WZ or ZB structure are shown in yellow. Therefore, the yellow atoms identify grain boundaries and possibly dislocations. Plane and crosssectional view images are provided for each simulated growth on the [0001] WZ and [111] ZB oriented substrates. The plane view images are located at $\sim 80 \AA$ and $\sim 100 \AA$ from the bottom of the substrate, for [0001] WZ and [111] ZB respectively, and show a quasi-hexagonal network of grain boundaries connecting $\mathrm{WZ}$ and ZB domains as typically observed in polycrystalline films [32]. The cross-sectional images showed that the domain boundaries are typically normal or with some angle, between $20^{\circ}$ to $35^{\circ}$, to the direction of growth. Both growths showed similar polytypism with a significant number of WZ and ZB domains. Similar CdS polytypism has been reported experimentally by Ouyang [21] and Kumar [5] in nanowires and quantum dots.

Table 1 summarizes and compares the results of this work with results reported for nanostructures in the literature. Even though the nanostructure formation has other factors that are not considered in our simulations, there is a similar trend of the defect types with respect to growth directions. This indicates that the substrate orientation is an important factor for single crystal growth.

The dislocations in the simulated growths on the [0001] WZ and [111] ZB substrates were analyzed using a dislocation extraction algorithm for ZB [33] [34] and WZ [35] lattices. Figure 4 shows color coded line representations of the dislocation networks. The dislocations were classified according to their ZB or WZ Burger's vector family. Seven different Burgers vectors were identified in the ZB and $W Z$ families. The Burger's vectors are listed and color coded in the legends of Figure 4. For clarity, separate images are provided for the ZB and WZ families of dislocations. Overall the dislocations are uniformly distributed within the overgrowths but no dislocations were detected in the substrates. By far the most prevalent dislocations are the Shockley partials with a Burger's vector of $1 / 6\langle 112\rangle$. Similar dislocations were identified experimentally by Li [36] in ZB CdTe. Full dislocations with a Burger's vector of $\langle 0001\rangle$ are also present with significant density, as shown in Figures 4 (e) to (h). Other dislocations as listed in the legend were also present in lower density.

These results indicate a general tendency in which homoepitaxy growth of CdS on substrate orientations with rectangular surface geometry lead to single crystal films. In contrast, growth on hexagonal geometry resulted in polytypism. In order to investigate whether this general tendency persists or the results could be attributed to other factors, growth simulations were performed at various temperatures and deposition rates on a Cd-terminated CdS[0001] substrate. Figure 5(a) plots the dislocation density at different temperatures. Here the dislocation density is defined as the summation of the total dislocation line length divided by the heterostructure's volume. While the dislocation density decreased with increasing temperature, polytypism persisted. Similarly Figure 5(b) shows that the deposition rate affected the dislocation density (at the higher temperature), but the dislocation density was not reduced to zero. Finally, in order to observe the effect of substrate termination, growth was simulated on a S-terminated CdS[0001] substrate as shown in the inset of Figure $5(b)$. In general the dislocation density was higher on the S-terminated substrate with polytypism 
persisting. The simulation results are in good agreement with literature data and indicate the same dependence of polytypism on substrate orientation. The existence of polytypism in the films grown on the hexagonal substrates is attributed to the small difference in the structural energy associated with the WZ and ZB phases [18]. Since both WZ and ZB phases contain the exact same hexagonal surface geometry and similar structural energy, it is equally favorable to nucleate either structure.

\section{Conclusions}

MD simulations of CdS homoepitaxial growth were performed using different substrates orientations. The substrates can be subdivided in two groups: non-polar rectangular and polar hexagonal surface geometries. In general the rectangular cases showed highly crystalline films with only point defects. The exception was the growth along [112] which showed a low density of stacking faults. In contrast the hexagonal cases displayed highly defected structures with presence of SF, twinning, and polytypism. A structural analysis was performed to identify the WZ and ZB domains and corresponding grain boundaries for the hexagonal growth. The dislocation analysis indicated that the most predominant dislocations are Shockley partials with a Burger's vector of $1 / 6\langle 112\rangle$ and full dislocations with a Burger's vector of $\langle 0001\rangle$ for the ZB and WZ families respectively. In general the simulation results agree well with experimental data reported in literature predicting defective and single crystal growths on hexagonal and rectangular surfaces respectively. Lastly, different deposition conditions were explored to improve the film's crystal quality for [0001] WZ case. The simulations showed that films grown at higher temperatures contained lower dislocation length densities. Low deposition rates and [0001] substrate polarity also decreased the film's dislocation length densities, although these effects were only observed at high deposition temperatures. Polytypsim observed in the films grown on the substrates with hexagonal surface geometry is attributed to the similar formation energies of the WZ and ZB phases.

\section{Acknowledgment}

Sandia National Laboratories is a multiprogram laboratory managed and operated by Sandia Corporation, a wholly owned subsidiary of Lockheed Martin Corporation, for the US Department of Energy's National Nuclear Security Administration under contract DE-AC04-94AL85000. This work was supported by the Department of Energy through the BRIDGE program (DE-EE0005958) and the Extreme Science and Engineering Discovery Environment (XSEDE), which is supported by National Science Foundation grant number $\mathrm{ACl}-1053575$.

\section{References}

[1] J. Britt and C. Ferekides, "Thin-film CdS/CdTe solar cell with 15.8\% efficiency," J. Appl. Phys., vol. 62, no. 22, pp. 2851-2852, 1993.

[2] K. Ramanathan, M. A. Contreras, C. L. Perkins, S. Asher, F. S. Hasoon, J. Keane, D. Young, M. Romero, W. Metzger, R. Noufi, J. Ward and A. Duda, "Properties of $19.2 \%$ efficiency ZnO/CdS/CulnGaSe2 thin-film solar cells," Prog. Photovolt: Res. Appl., vol. 11, no. 4, pp. 225-230, 2003.

[3] K. Senthil, D. Mangalaraj, S. K. Narayandass, B. Hong, Y. Roh, C. S. Park and J. Yi, "Argon and nitrogen implantation effects on the structural and optical properties of vacuum evaporated cadmium sulphide thin films," Semicond. Sci. Technol., vol. 17, no. 2, pp. 97-103, 2002.

[4] X. Wu and Y. Tao, "Growth of CdS nanowires by physical vapor deposition," J. Crystal Growth, vol. 242, no. 3, pp. 309-312, 2002.

[5] P. Kumar, NupurSaxena, F.Singh and A. Agarwal, "Nanotwinning in CdS quantum dots," Physica B: Condensed Matter, vol. 407, no. 17, pp. 3347-3351, 2012.

[6] O. Hayden, A. B. Greytak and D. C. Bell, "Core-shell nanowire light-emitting diodes," Adv. Mater., 
vol. 17, no. 6, pp. 701-704, 2005.

[7] N. Kouklin, L. Menon, A. Z. Wong, D. W. Thompson, J. A. Woollam, P. F. Williams and S. Bandyopadhyay, "Giant photoresistivity and optically controlled switching in self-assembled nanowires," Appl. Phys. Lett., vol. 79, no. 26, pp. 4423-4425, 2001.

[8] X. Duan, Y. Huang, Y. Cui, J. Wang and C. M. Lieber, "Indium phosphide nanowires as building blocks for nanoscale electronic and optoelectronic devices," Nature, vol. 409, no. 6816, pp. 66-69, 2001.

[9] C. J. Barrelet, A. B. Greytak and C. M. Lieber, "Nanowire photonic circuit elements," Nano Letters, vol. 4, no. 10, pp. 1981-1985, 2004.

[10] R.-M. Ma, L. Dai, H.-B. Huo, W.-J. Xu and G. G. Qin, "High-performance logic circuits constructed on single CdS nanowires," Nano letters, vol. 7, no. 11, pp. 3300-3304, 2007.

[11] X. Duan, C. Niu, V. Sahi, J. Chen, J. W. Parce, S. Empedocles and J. L. Goldman, "High-performance thin-film transistors using semiconductor nanowires and nanoribbons," Nature, vol. 425, no. 6955, pp. 274-278, 2003.

[12] T. Zhai, X. Fang, Y. Bando, Q. Liao, X. Xu, H. Zeng, Y. Ma, J. Yao and D. Golberg, "Morphologydependent stimulated emission and field emission of ordered CdS nanostructure arrays," Acs Nano, vol. 3, no. 4, pp. 949-959, 2009.

[13] H. Dang, V. Singh, S. Rajaputra, S. Guduru, J. Chen and B. Nadimpally, "Cadmium sulfide nanowire arrays for window layer applications in solar cells," Solar Energy Materials and Solar Cells, vol. 126, pp. 184-191, 2014.

[14] L. Li, S. Yang, X. Zhang, L. Wang, Z. Jiang, Q. Lin, C. Wang, F. Han and N. Peng, "Single CdS nanowire photodetector fabricated by FIB," Microelectronic Engineering, vol. 126, pp. 27-30.

[15] D. Mo, J. Liu, H. Yao, J. Duan, M. Hou, Y. Sun, Y. Chen, Z. Xue and L. Zhang, "Preparation and characterization of $\mathrm{CdS}$ nanotubes and nanowires by electrochemical synthesis in ion-track templates," Journal of Crystal Growth, vol. 310, no. 3, pp. 612-616, 2008.

[16] J. Schaffner, M. Motzko, A. Tueschen, A. Swirschuk, H.-J. Schimpe, A. Klein, T. Modes, O. Zywitzki and W. Jaegermann, "12\% efficient CdTe/CdS thin film solar cells deposited by low-temperature close space sublimation," J. Appl. Phys, vol. 110, no. 6, p. 064508, 2011.

[17] M. Sun, W. Fu, Q. Li, G. Yin, K. Chi, X. Zhou, J. Ma, L. Yang, Y. Mu, Y. Chen and H. Yang, "Facile fabrication of CdS nanorod arrays on the transparent conducting substrates and their photoelectrochemical properties," Journal of crystal growth, vol. 377, pp. 112-117, 2013.

[18] C.-Y. Yeh, Z. W. Lu, S. Froyen and A. Zunger, "Zinc-blende -wurtzite polytypism in semiconductors," Physical Review B, vol. 46, no. 16, p. 10086, 1992.

[19] J. Zhang, Y. Yang, F. Jiang, J. Li, B. Xu, S. Wang and X. Wang, "Fabrication of semiconductor CdS hierarchical nanostructures," J. crystal growth, vol. 293, no. 2, pp. 236-241, 2006.

[20] W. Liu, C. Jia, C. Jin, L. Yao, W. Cai and X. Li, "Growth mechanism and photoluminescence of CdS nanobelts on Si substrate," J. Crystal Growth, vol. 269, no. 2, pp. 304-309, 2004.

[21] L. Ouyang, K. N. Maher, C. L. Yu, J. McCarty and H. Park, "Catalyst-assisted solution-liquid-solid synthesis of CdS/CdSe nanorod heterostructures," Journal of the American Chemical Society, vol. 129, no. 1, pp. 1133-138, 2007.

[22] D. Routkevitch, T. Bigioni, M. Moskovits and J. M. Xu, "Electrochemical fabrication of CdS nanowire arrays in porous anodic aluminum oxide templates," The Journal of Physical Chemistry, vol. 100, no. 33, pp. 14037-14047, 1996.

[23] K. S. Ramaiah, R. Pilkington, A. Hill, R. Tomlinson and A. Bhatnagar, "Structural and optical investigations on CdS thin films grown by chemical bath technique," Materials Chemistry and Physics, vol. 68, no. 1, pp. 22-30, 2001.

[24] D. J. Milliron, S. M. Hughes, Y. Cui, L. Manna, J. Li, L.-W. Wang and A. P. Alivisatos, "Colloidal nanocrystal heterostructures with linear and branched topology," Nature, vol. 430, no. 6996, pp. 
190-195, 2004.

[25] C. J. Barrelet, Y. Wu, D. C. Bell and C. M. Lieber, "Synthesis of CdS and ZnS nanowires using singlesource molecular precursors," Journal of the American Chemical Society, vol. 125, no. 38, pp. 11498-11499, 2003.

[26] R. M. Ma, L. Dai and G. G. Qin, "Enhancement-mode metal-semiconductor field-effect transistors based on single n-CdS nanowires," Applied Physics Letters, vol. 90, no. 9, p. 3109, 2007.

[27] S. Plimpton, "Fast Parallel Algorithms for Short-Range Molecular Dynamics," J. Comp. Phys, vol. 117, pp. 1-19, 1995.

[28] X. W. Zhou, D. K. Ward, J. E. Martin, F. B. v. Swol, J. L. Cruz-Campa and D. Zubia, "Stillinger-Weber potential for the II-VI elements Zn-Cd-Hg-S-Se-Te," Phys. Rev. B, vol. 88, no. 8, p. 085309, 2013.

[29] W. G. Hoover, "Canonical dynamics: Equilibrium phase-space distributions," Phys. Rev. A, vol. 31, no. 3, p. 1695, Mar 1985.

[30] A. Stukowski, "Visualization and analysis of atomistic simulation data with OVITO - the Open Visualization Tool," Modelling Simul. Mater. Sci. Eng., vol. 18, no. 015012, 2010.

[31] H.-J. Gossmann, L. C. Feldman and W. M. Gibson, "Reordering of reconstructed Si surfaces upon Ge deposition at room temperature," Physical review letters, vol. 53, no. 3, p. 294, 1984.

[32] H. Moutinho, J. Moseley, M. Romero, R. Dhere, C.-S. Jiang, K. Jones, J. Duenow, Y. Yan and M. AlJassim, "Grain boundary character and recombination properties in CdTe thin films," Photovoltaic Specialists Conference (PVSC), vol. 2013 IEEE 39th, pp. 3249-3254, 2013.

[33] A. Stukowski and K. Albe, "Extracting dislocations and non-dislocation crystal defects from atomistic simulation data," Modelling Simul. Mater. Sci. Eng., vol. 18, no. 085001, 2010.

[34] A. Stukowski, V. V. Bulatov and A. Arsenlis, "Automated identification and indexing of dislocations in crystal interfaces," Modelling and Simulation in Materials Science and Engineering, vol. 20, no. 085007, p. 16, 2012.

[35] J. J. Chavez, "Molecular Dynamics Study on Defect Reduction Strategies Towards the Fabrication of High Performance Cd1-xZnxTe/CdS Solar Cells," In Press, El Paso, TX, 2015.

[36] C. Li, J. Poplawsky, Y. Wu, A. R. Lupini, A. Mouti, D. N. Leonard, N. Paudel, K. Jones, W. Yin, M. AlJassim, Y. Yan and S. J. Pennycook, "From atomic structure to photovoltaic properties in CdT esolar cells," Ultramicroscopy, vol. 134, pp. 113-125, 2013. 


\section{Figures}

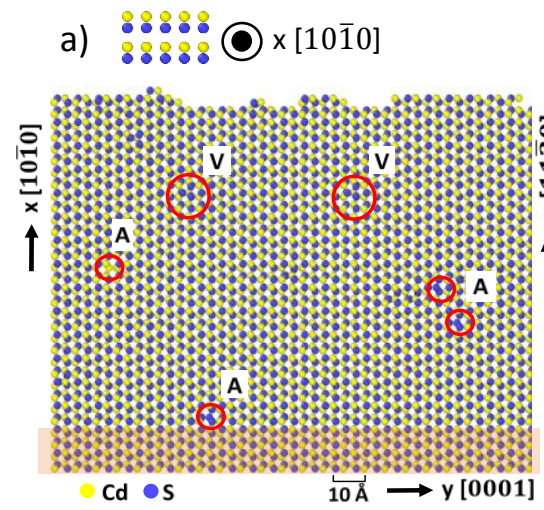

$$
\text { b }: \%
$$

c) $\because: \because \bigcirc \times[110]$
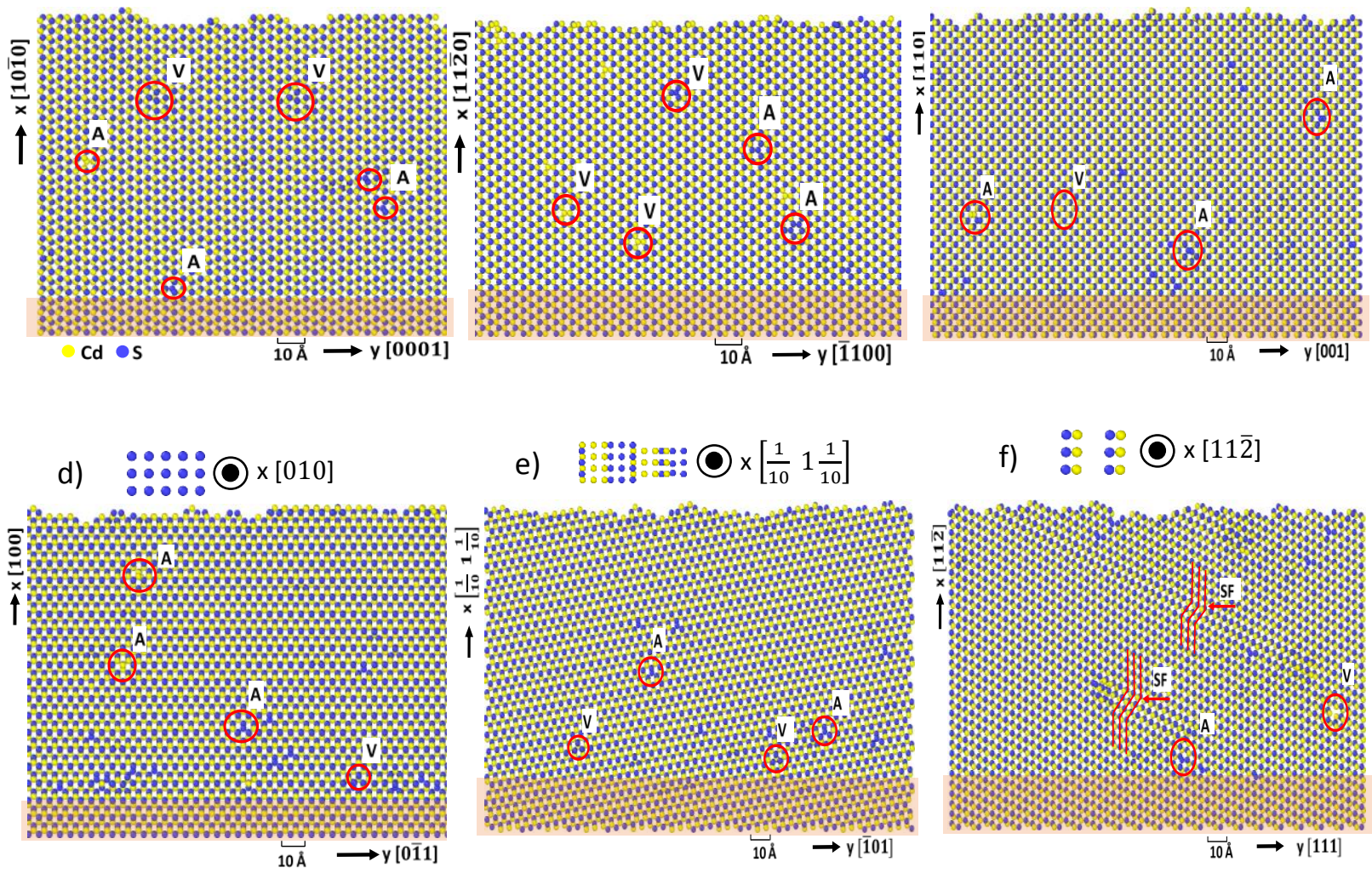

e)

f) $\because \because 0 \times[11 \overline{2}]$
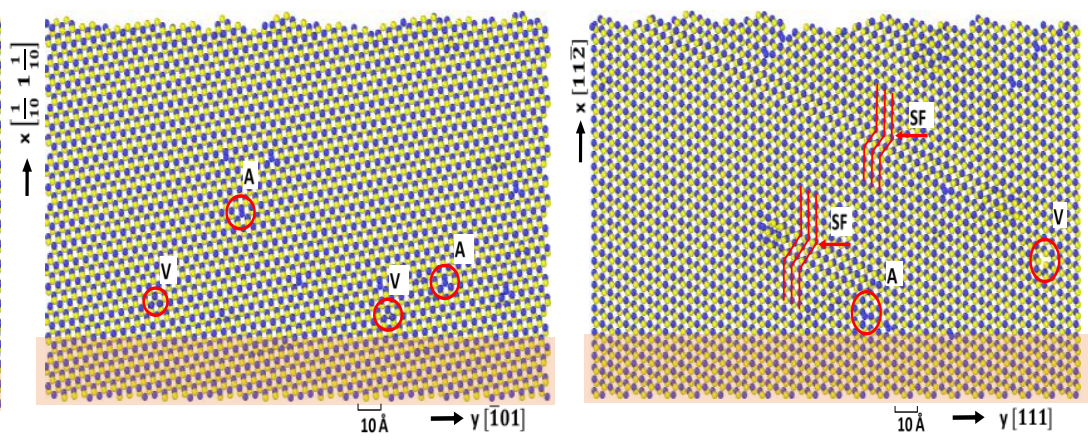

Figure 1. Atomistic visualizations of MD simulations for $\mathrm{CdS}$ homoepitaxial growth along (a) [1010] $W Z,(b)[11 \overline{2} 0] W Z,(c)[110] Z B,(d)[010] Z B,(e)\left[\frac{1}{10} 1 \frac{1}{10}\right] Z B$ and (f) $[11 \overline{2}] Z B$, directions. Shaded regions represent the initial substrate.

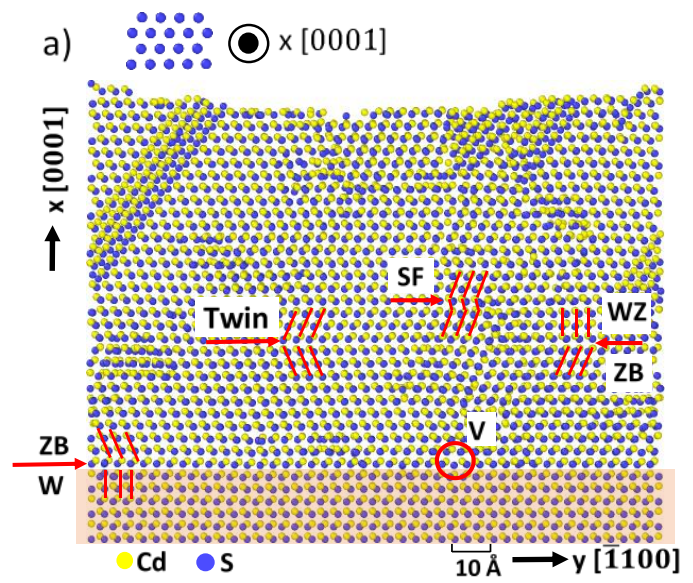

b) $\quad \because \because \because 0 \times[111]$

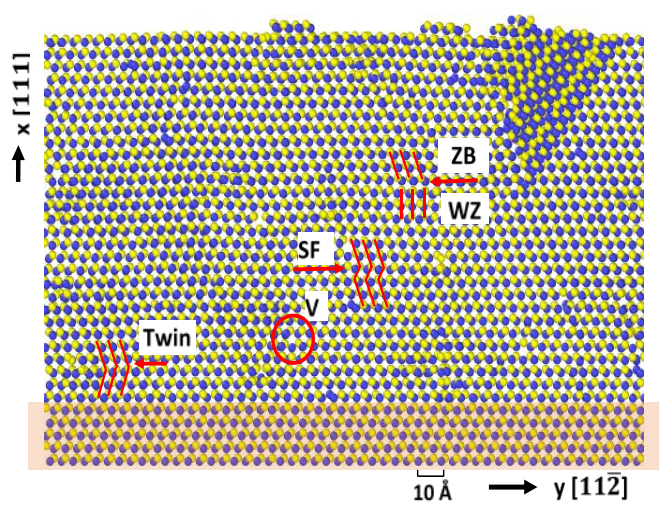

Figure 2. Atomistic visualizations of MD simulations for CdS homoepitaxial growth along (a) [0001] WZ (b) [111] ZB, directions. Shaded regions represent the initial substrate. 
(a)

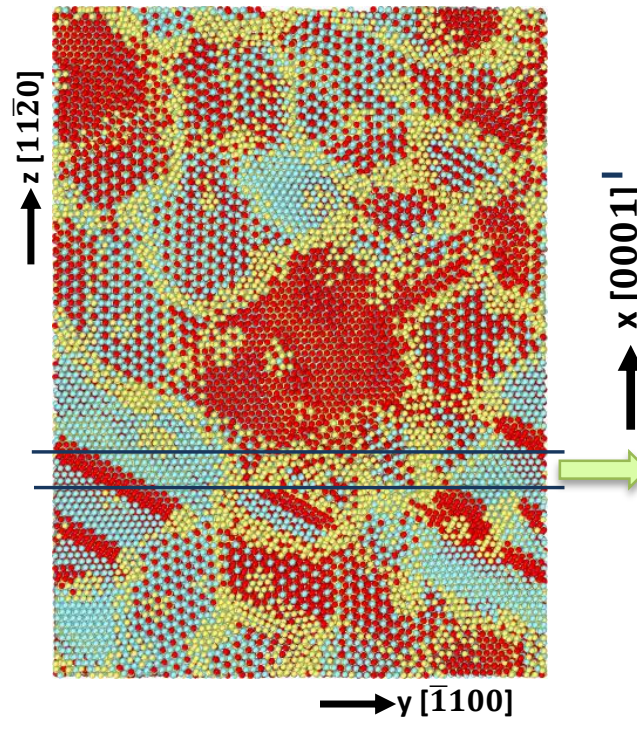

(c)

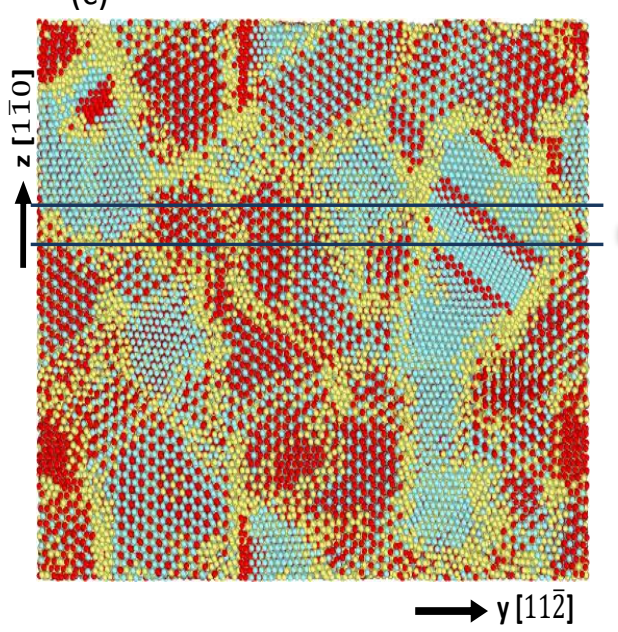

(b)

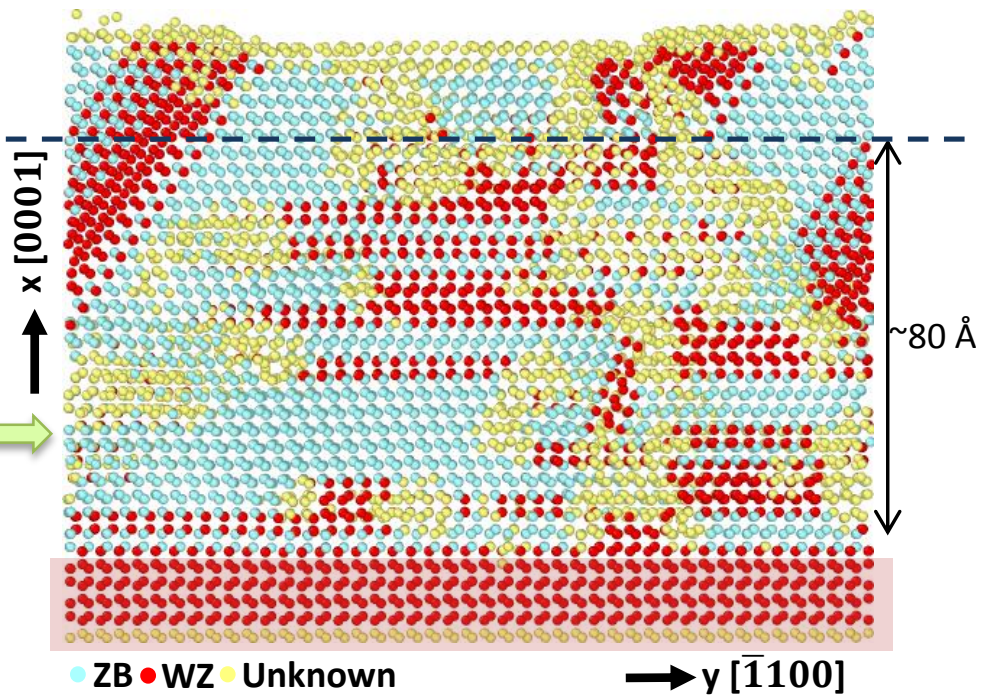

(d)

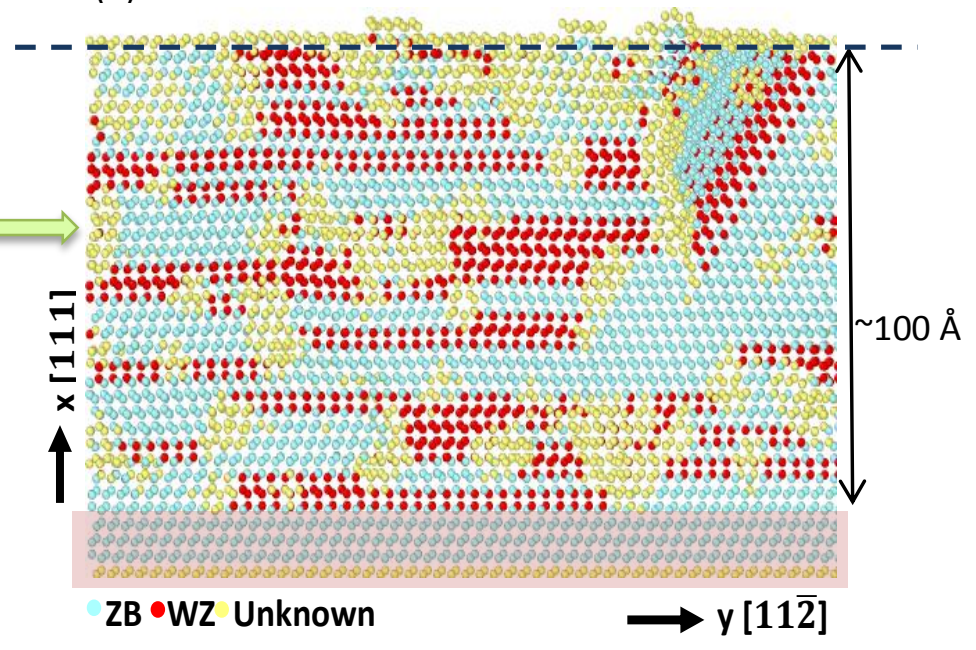

Figure 3. Structural maps of CdS homoepitaxial growths on hexagonal surfaces. (a) Top and (b) crosssectional views of CdS grown on [0001] WZ. (c) Top and (d) cross-sectional views of CdS grown on [111] $\mathrm{ZB}$, shaded regions represent the initial substrate. 
(a)

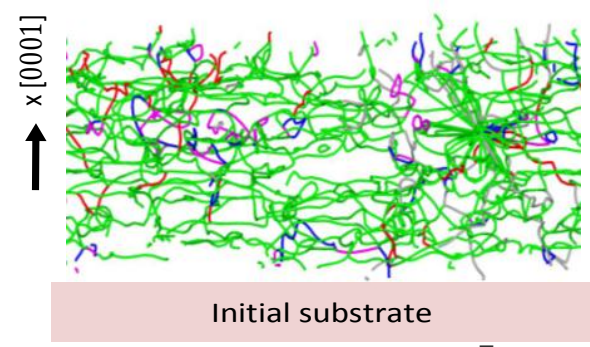

(c)

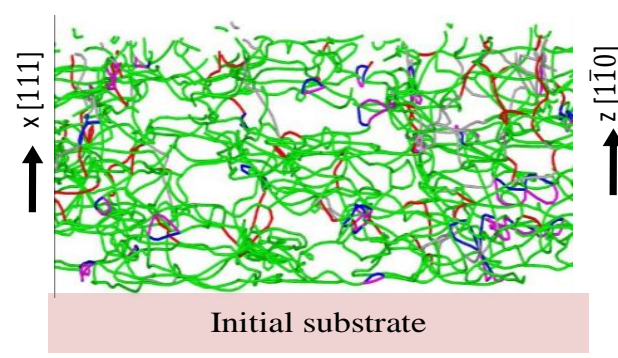

(e) (b)

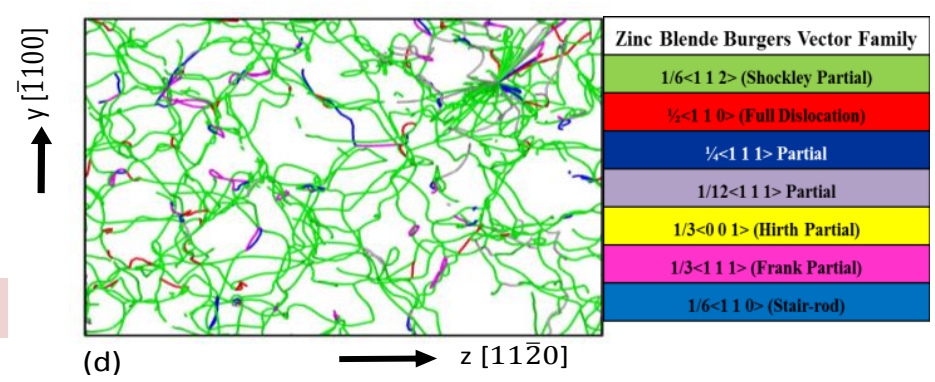

(d)
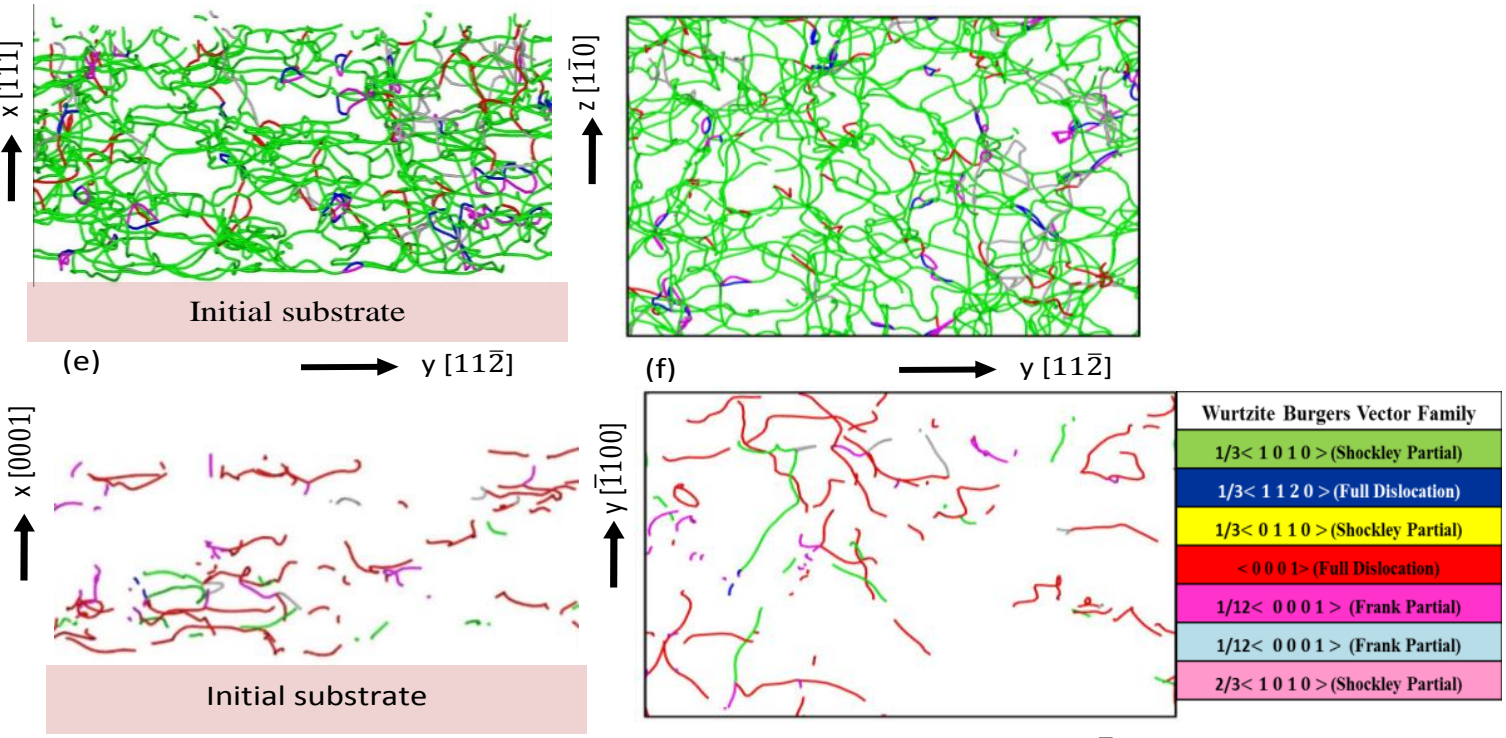

(g)

$y[\overline{1} 100]$

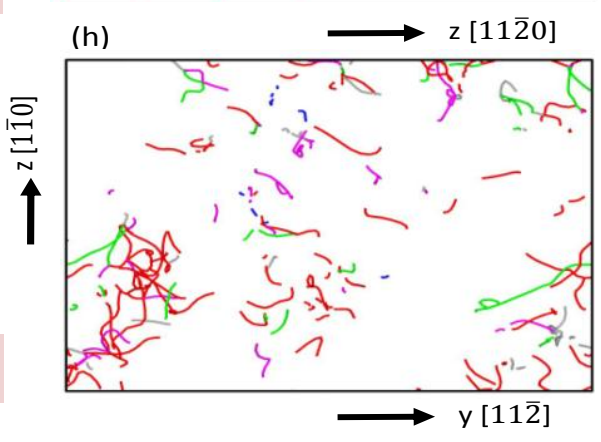

Figure 4. Cross sectional (left column) and top view (right column) visualization of dislocations in the films grown on the [0001] WZ and [111] ZB CdS substrates are shown. The images are grouped according to either ZB or WZ Burger's vector types as indicated by the color legends. (a), (b), (e) and (f) are dislocations in the CdS grown on [0001]CdS. (c), (d), (g), and (h) are dislocations in the CdS grown on [111]CdS. 

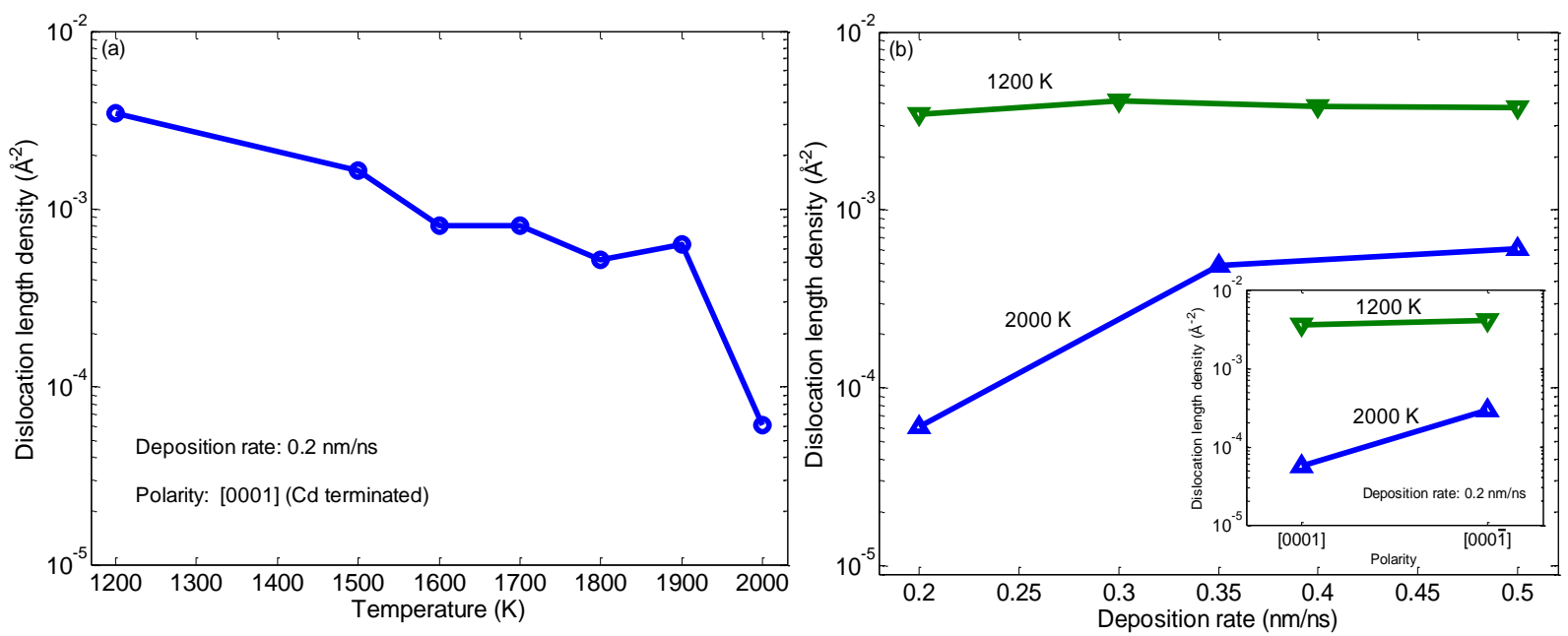

Figure 5. Dislocation length density for (a) different temperatures at constant deposition rate and (b) different deposition rates at $1200 \mathrm{~K}$ and $2000 \mathrm{~K}$. The inset is the dislocation length density for different polarities: [0001] and [0001] are Cd and S terminated respectively, at $1200 \mathrm{~K}$ and $2000 \mathrm{~K}$.

Table 1. Comparison of our simulated results to experimental results reported in the literature.

\begin{tabular}{|c|c|c|c|c|c|}
\hline $\begin{array}{l}\text { Growth } \\
\text { direction }\end{array}$ & Deposition Method & Crystallinity & Structures & Defect type & Ref \\
\hline \multirow{8}{*}{ WZ [0001] } & MD Simulations & Polycrystalline & & $\begin{array}{l}\text { SF, Twinning, } \\
\text { polytypism }\end{array}$ & This work \\
\hline & Electrochemical & Polycrystalline & Nanowires & $\begin{array}{l}\text { Grain } \\
\text { boundaries }\end{array}$ & {$[22]$} \\
\hline & $\begin{array}{l}\text { Chemical Bath } \\
\text { Deposition }\end{array}$ & Polycrystalline & Nanorods & $\begin{array}{l}\text { SF, } \\
\text { polytypism }\end{array}$ & {$[21]$} \\
\hline & $\begin{array}{l}\text { Chemical Bath } \\
\text { Deposition }\end{array}$ & Polycrystalline & Nanorods & $\begin{array}{l}\text { SF, } \\
\text { polytypism }\end{array}$ & [24] \\
\hline & $\begin{array}{l}\text { Chemical Bath } \\
\text { Deposition }\end{array}$ & Polycrystalline & & $\begin{array}{l}\text { Screw, Hirth } \\
\text { dislocation }\end{array}$ & [23] \\
\hline & MOCVD & Single crystal & $\begin{array}{l}\text { Nanorods, } \\
\text { Nanowires }\end{array}$ & & [12] \\
\hline & Thermal evaporation & Single crystal & $\begin{array}{l}\text { Nanorods, } \\
\text { nanoneedles }\end{array}$ & & [19] \\
\hline & Thermal evaporation & Single crystal & Nanobelts & & {$[20]$} \\
\hline \multirow{2}{*}{ ZB $[111]$} & MD Simulations & Polycrystalline & & $\begin{array}{l}\text { SF, Twinning, } \\
\text { polytypism }\end{array}$ & This work \\
\hline & $\begin{array}{l}\text { chemical co- } \\
\text { precipitation }\end{array}$ & Polycrystalline & $\begin{array}{l}\text { Quantum } \\
\text { Dots }\end{array}$ & $\begin{array}{l}\text { SF, Twinning, } \\
\text { polytypism }\end{array}$ & [5] \\
\hline WZ [10̄̄o] & $\begin{array}{l}\text { MD Simulations } \\
\text { CVD }\end{array}$ & $\begin{array}{l}\text { Single crystal } \\
\text { Single crystal }\end{array}$ & Nanowires & & $\begin{array}{l}\text { This work } \\
\text { [25] }\end{array}$ \\
\hline \multirow{3}{*}{ WZ [11̄̄0] } & MD Simulations & Single crystal & & & This work \\
\hline & CVD & Single crystal & Nanowires & & [26] \\
\hline & CVD & Single crystal & Nanowires & & {$[25]$} \\
\hline ZB [110] & MD Simulations & Single crystal & & & This work \\
\hline ZB [010] & MD Simulations & Single crystal & & & This work \\
\hline ZB $\left[\frac{1}{10} 1 \frac{1}{10}\right]$ & MD Simulations & Single crystal & & & This work \\
\hline $\mathrm{ZB}[112]$ & MD Simulations & Single-crystal & & SF & This work \\
\hline
\end{tabular}

